\title{
Use of 3D Parametric Models in the Automotive Component Design Process
}

\author{
Michal Fabian ${ }^{1 *}$, František Kupec ${ }^{1}$ \\ 1 Faculty of Mechanical Engineering, Technical University of Košice, Mäsiarska 74, Košice, 040 01, Slovakia \\ *Corresponding author's email: michal.fabian@tuke.sk
}

\begin{abstract}
The process of design, development and production of cars has been strongly influenced by the use of computer technology since the 1980s. The philosophy of CAD (Computer Aided Design) and CAM (Computer Aided Manufacturing) approaches has become an integral part of the automotive industry. These technologies have shortened the innovation cycle in car model lines to 6-8 years. In the middle of this cycle, the so-called facelift of the model line is employed, when there are certain shape changes of the exterior and interior of the car or the equipment is expanded with new or improved elements. CAx systems enable changes to be made in such a short period of time. This article deals with the design of a fully parametric CAD model of a car interior element. Specifically, it is part of the door pull handle. The advantages of connecting a fully parametric model to a spreadsheet were pointed out. This link has a significant impact on the speed and flexibility of change processes on product models. The model was created in the Part Design volume modeler in the CAD system CATIA V5 Release21, which is used mainly in the automotive and aerospace industries. The parameters of the model were linked to the MS Excel spreadsheet.
\end{abstract}

Keywords: CAD, CAM, parametric modeling, 3D printing.

\section{INTRODUCTION}

The issue of Advanced CAD methods in automotive development was dealt with in [1]. The use of CAx approaches in the automotive development process is discussed in [2]. The importance of the virtual car model in the car concept development phase in automotive is described in [3].

Owing to the massive application of CAx systems, the development time of the car has been reduced to 48 months, or even less. The development process undergoes many phases of change, which are often repeated until the functional or aesthetic requirements are fulfilled. One of the initial stages of development is the assignment of the so-called "Package" (Fig. 1), which determines the basic dimensional requirements of a new car. The decisive dimensions are the wheelbase and the track between the wheels, from which the other dimensions of the car are derived. These dimensions classify the car into a class or category. Other important attributes are the choice of car drive unit, type of wheel suspension, transmission of traction to the road, etc. Everything was solved with respect to functionality, ergonomics, safety, ecology, and economy in [4]. The issue of production and assembly was addressed in [5] and [6]. Looking to the future of autonomous driving was dealt with in [7].

\section{CREATING A 3D PARAMETRIC MODEL OF THE PLASTIC COVER DOOR PULL HANDLE}

We discuss the importance of using the control parameters of the 3D model in the process of adjusting the shape and wall thickness of this handle cover (Fig. 2) manufactured as a plastic mold.

Before designing the cover, the parameters were defined. The length of the door pull handle can be modified by changing the value of the parameter representing the length dimension "Rear length", the total depth of the body by changing 


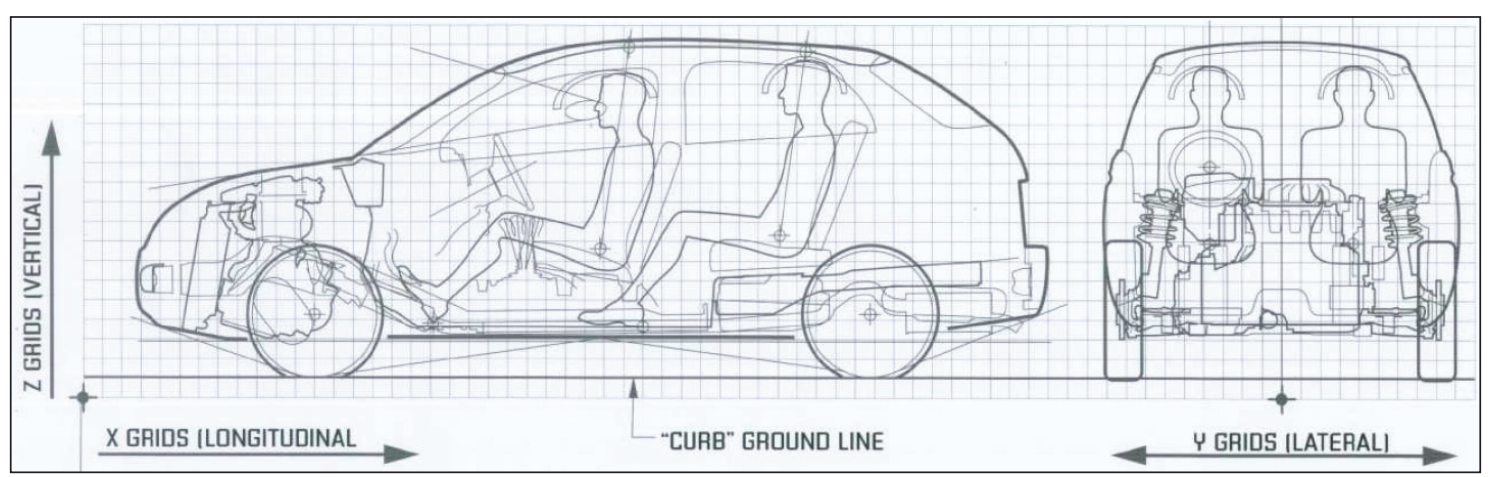

Fig. 1. Package - starting point for entering car requirements [4]

the value of parameter "Outside length", the depth of the handle for changing the handle by changing the Thickness value. The defined parameters are shown in Figure 3. The name and assignment of parameters to individual dimensions are defined in the Formula Editor improvement (Fig. 4).

The parameters defined in this way were assigned to specific values of parameterized distances in the profile sketch. The dimension values that
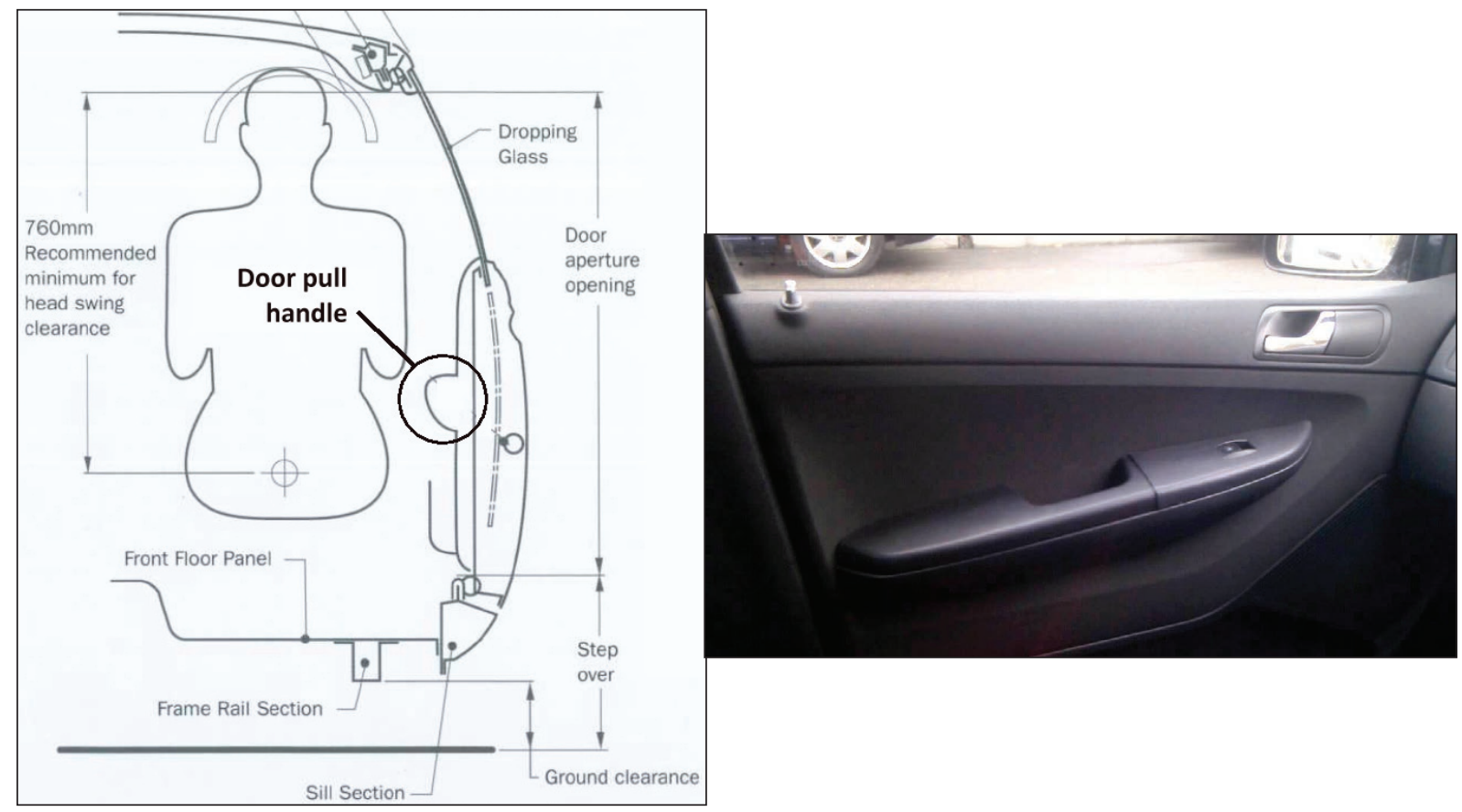

Fig. 2. Door pull handle
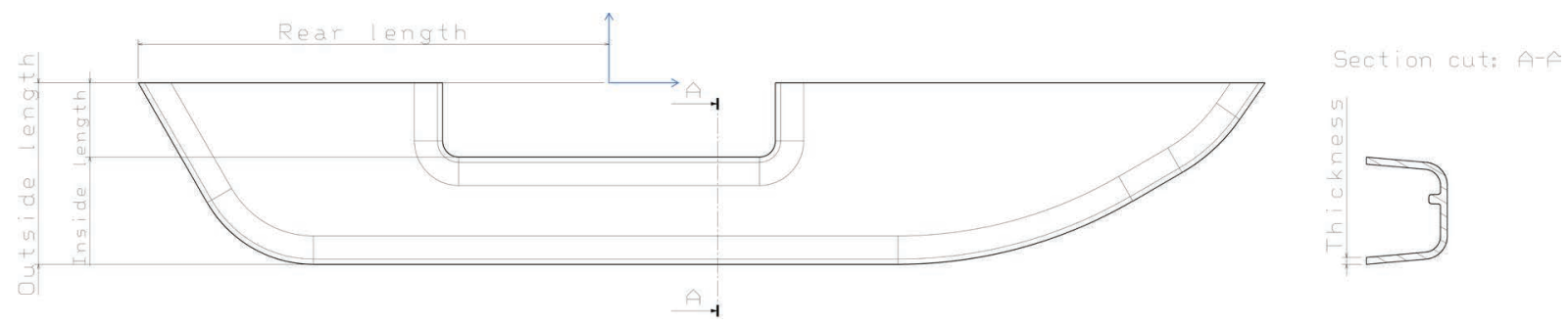

Fig. 3. Drawing with control parameter dimensions 


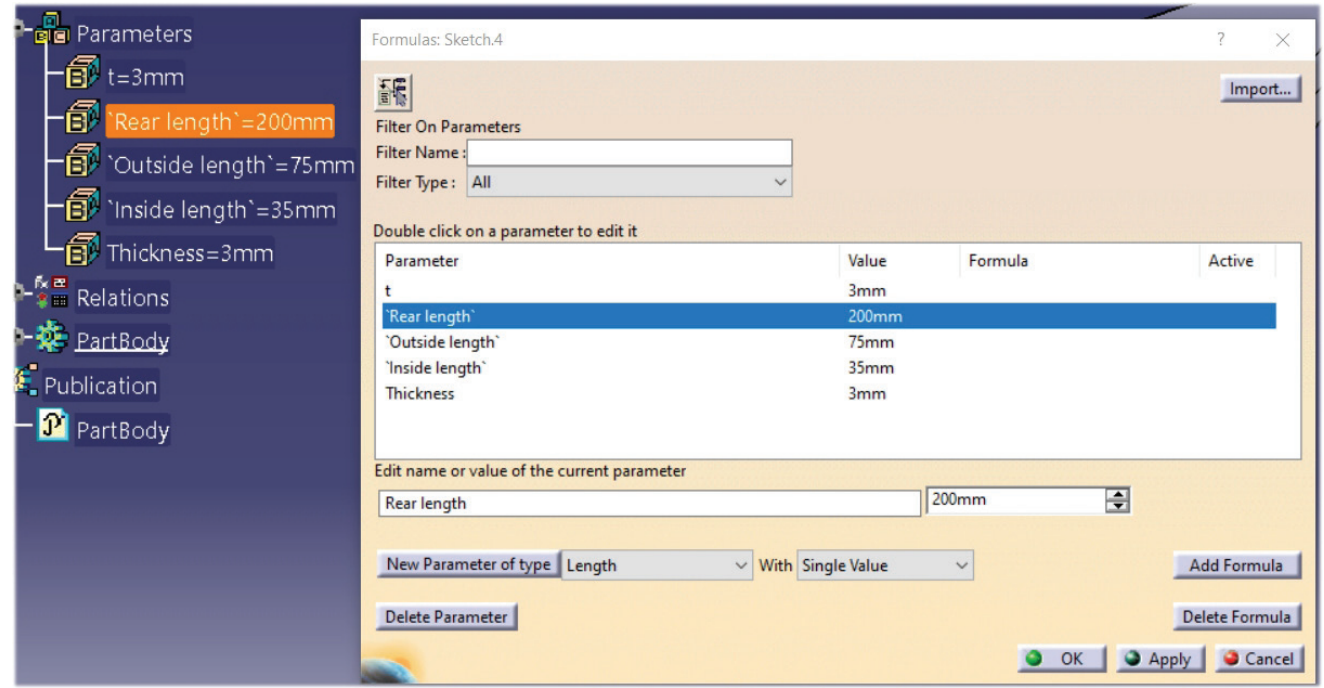

Fig. 4. Definition of parameters in "Formulas"

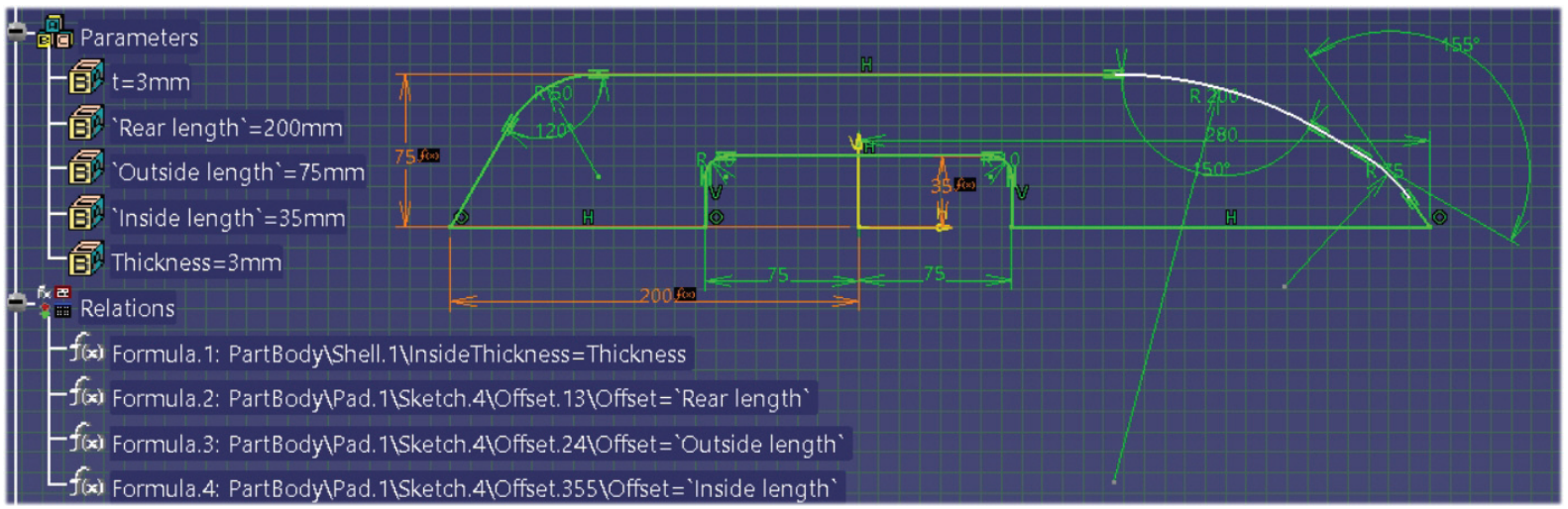

Fig. 5. Parameters and Relations

were only changeable in a particular Sketch become variables that can be changed at any time by changing a parameter value in the product tree without having to enter the Sketcher environment. In the following part, we will show how these Parameters can be linked to a spreadsheet and control the model using the values entered in the spreadsheet. By assigning the name of the parameter of the parameterized dimension, the so-called Relations, which are also shown in the product tree (Fig. 5).

In the Constraint Definition window (Fig. 6), the numerical value of the "dimensioned length" parameter of $200 \mathrm{~mm}$ is replaced by the predefined length parameter "Rear length” (Fig. 6). Selecting the $\mathrm{f}(\mathrm{x})$ icon edits the Formula Editor dialog box. In the top line of the Formula editor is the left side of the equation representing the CAD interpretation of the defined dimension, to which we assign the right side of the equation that will be represented by the length parameter "Rear Length” (Fig. 7).
Analogously, the "Outside length" and "Inside length" parameters were assigned to the numerically defined values of the sketch dimensions. The "Thickness" parameter assignment was defined in the Edit Parameter dialog box of the Shell command (Fig. 8). Subsequently, in the Formula editor, the CAD interpretation of the dimension was assigned the "Thickness" parameter, which

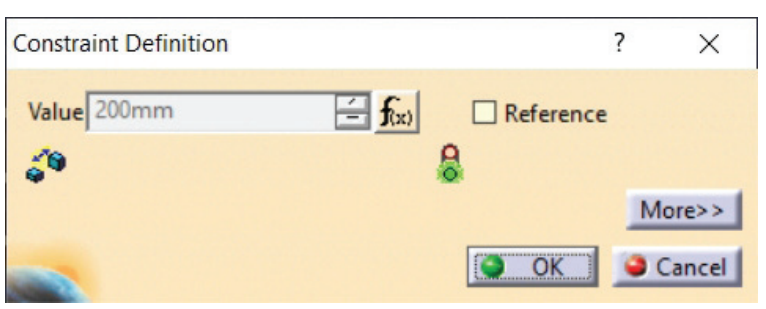

Fig. 6. Assigning a "Rear length" Constraint to a dimension 


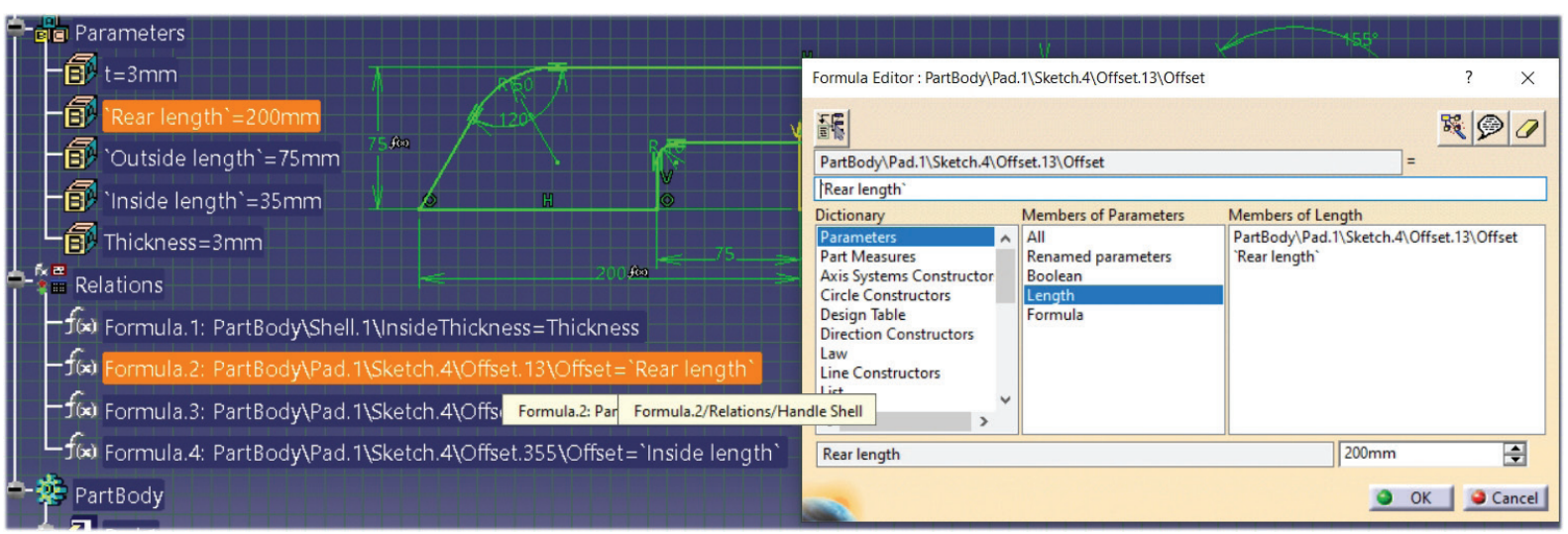

Fig. 7. Equation of a defined "Rear length" relation

represents the wall thickness of the molding (Fig. 9). Four controlled parameters described by formulas (1-4) determine the variable geometry of the plastic mold (Fig. 3).

$$
\text { PartBody } \backslash \text { Pad.1 } \backslash \text { Sketch.4 } \backslash \text { Offset.13 } \backslash \text { Offset }=\text { Rear length }
$$

PartBody $\backslash$ Pad.1 $\backslash$ Sketch.4\Offset.24\Offset = Outside length

PartBody $\backslash$ Pad.1 $\backslash$ Sketch.4\Offset.355 \Offset = Inside length

$$
\text { PartBody } \backslash \text { Shell.1 } \backslash \text { InsideThickness }=\text { Thickness }
$$
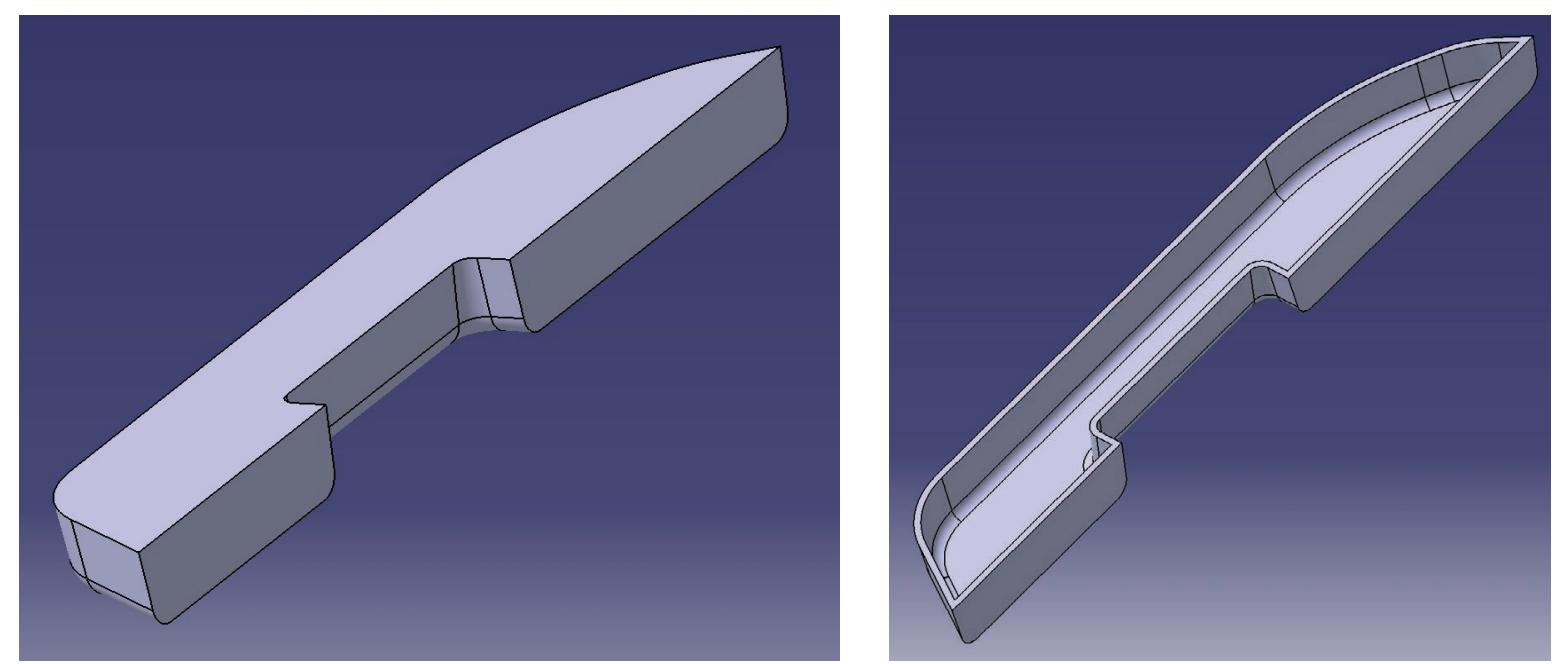

Fig. 8. Creating a handle and shell body

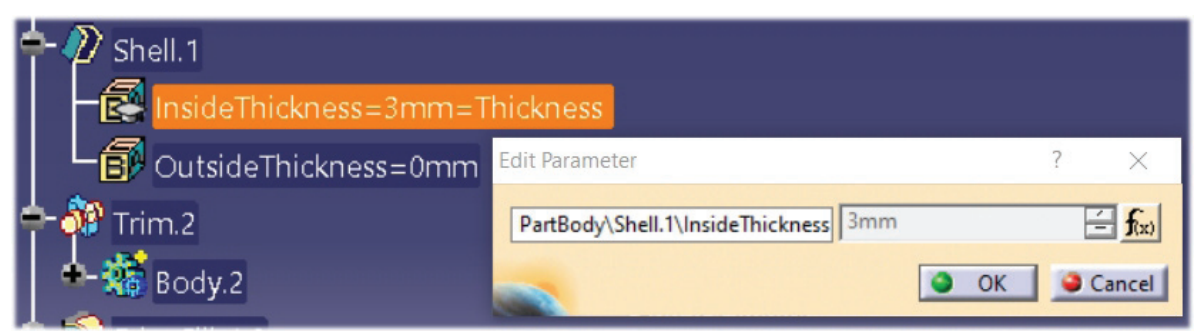

Fig. 9. Assigning the "Thickness" Constraint parameter to the Shell function 


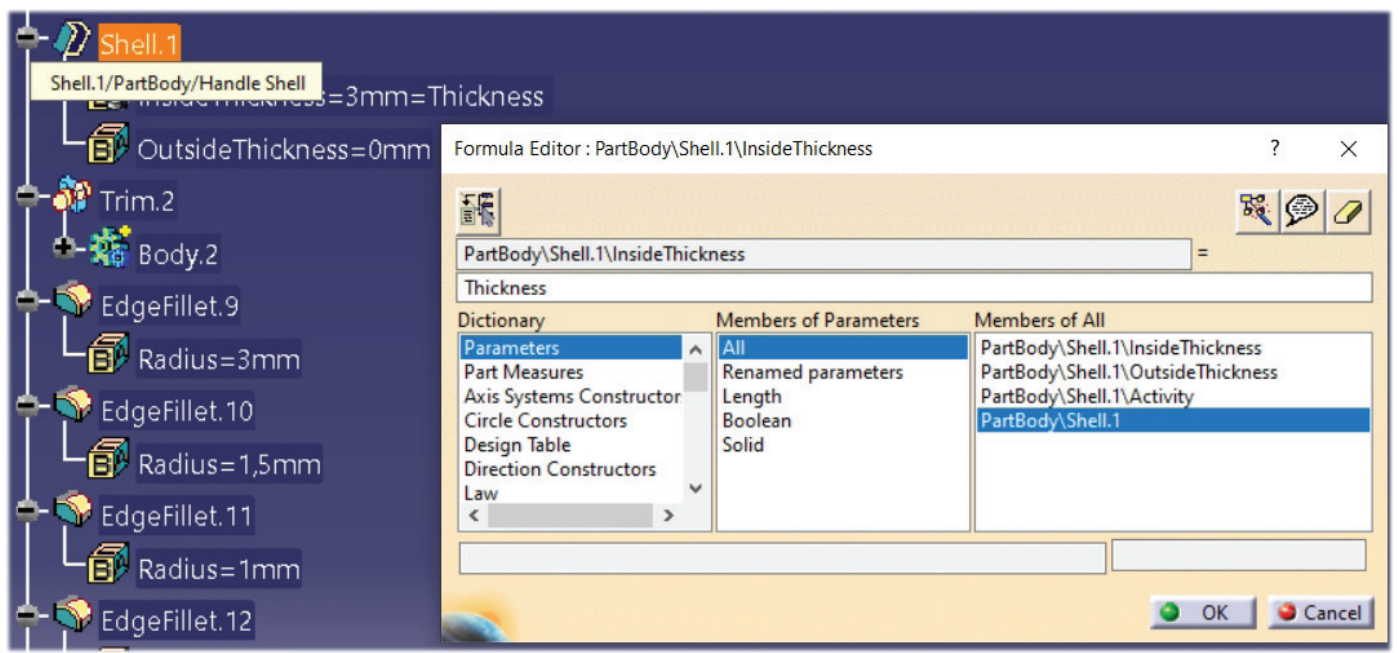

Fig. 10. The equation defining the "Thickness" relation

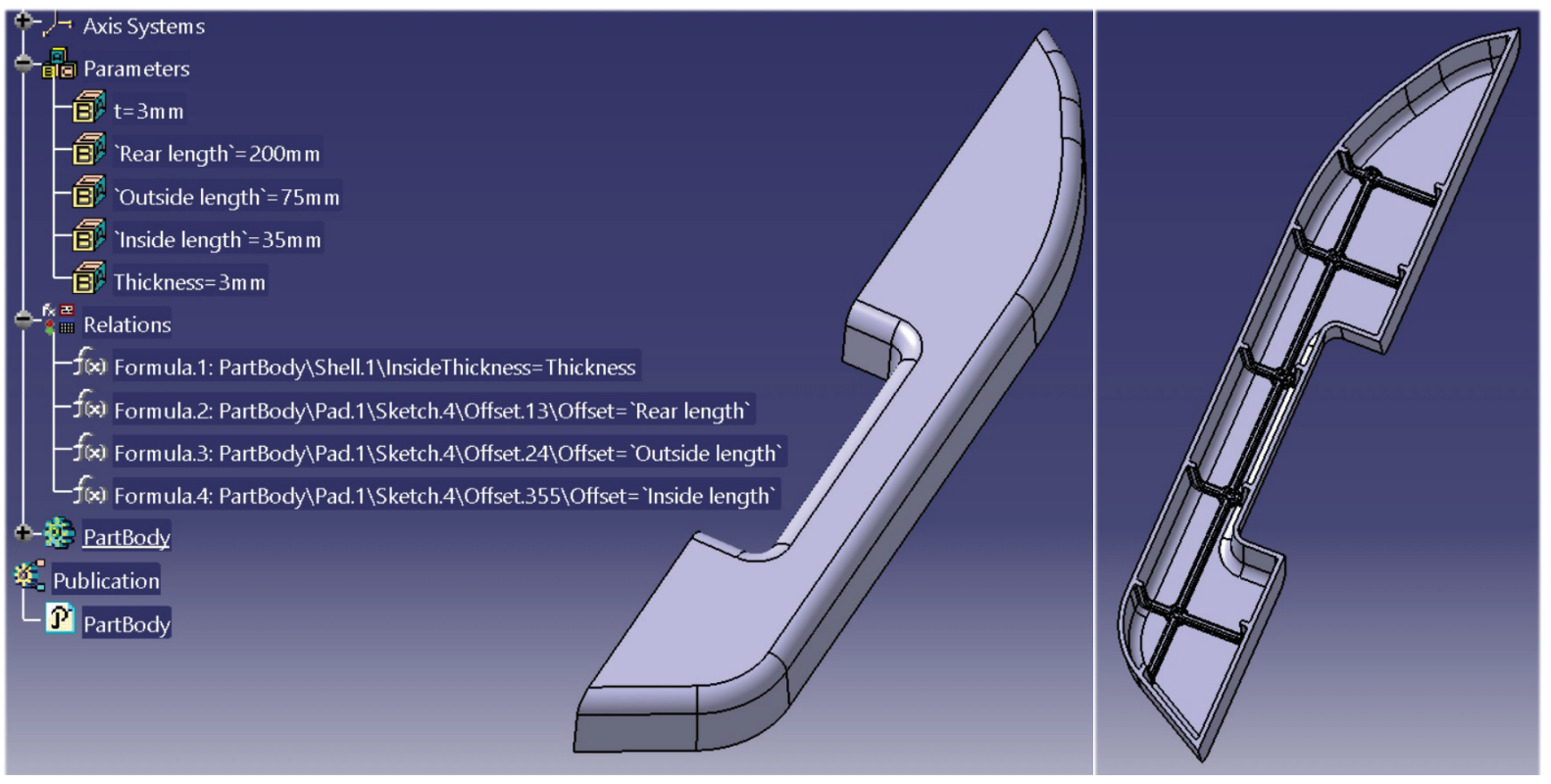

Fig. 11. Door pull handle cover with displayed Parameters and Relations in the Product tree

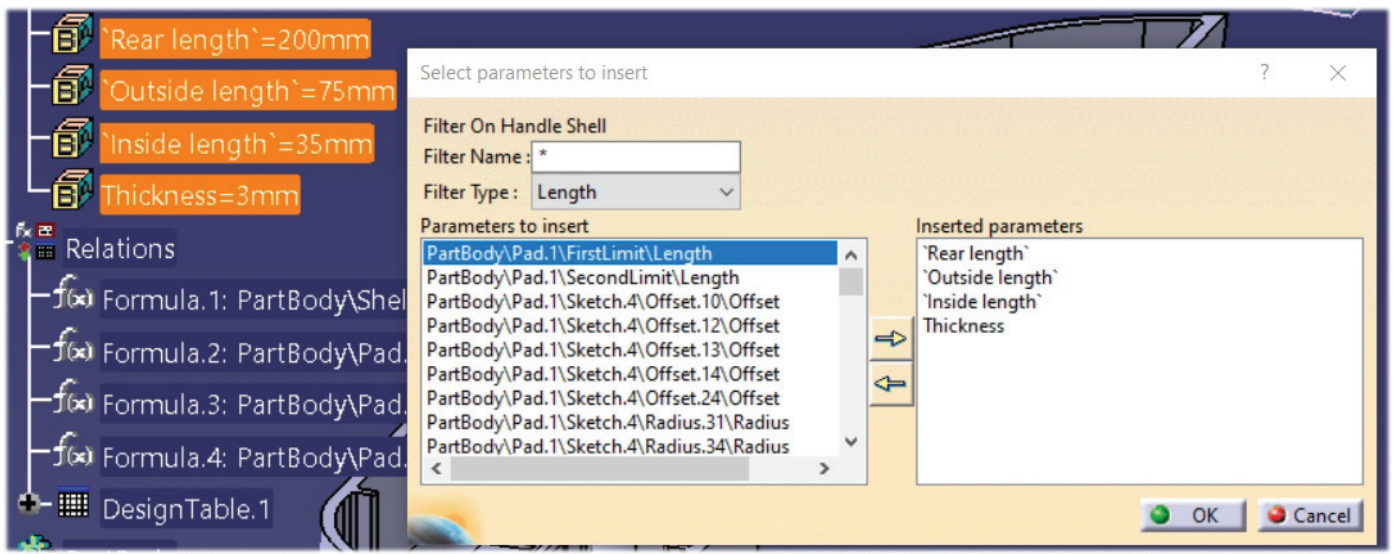

Fig. 12. Select Parameters to Control Model 


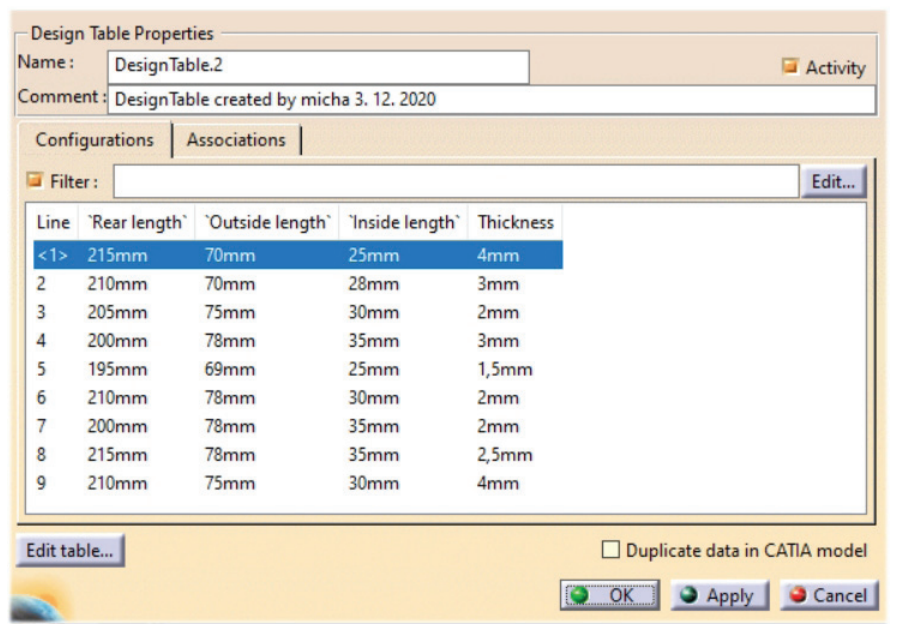

Fig. 13. Parameter values in MS Excel and in CATIA Design Table

A 3D model of Door pull handle cover (Fig. 10) was created in the CATIA V5solid and surface modeler software. The model was created regarding the manufacturability. When designing the component, rules for the design of plastic molds were applied. The requirements for a constant thickness of the wall of the mold, radiuses and bevels were also applied.

\section{MODEL CONTROL BY MS EXCEL}

At first we select Creation of a Design Table command and Defining model control parameters which will be linking to MS Excel. The selected parameters have been defined in order to control the dimensions of the model geometry (Fig. 11).

This step redirects us to the MS Excel environment, where we have the option to add values to individual parameters. This creates a dimensional database for the model (Fig. 12). The dimension database can be modified at any time. The model can then be controlled in a spreadsheet by parameter values (Fig. 13). Two different shapes for setting different parameter values in MS Excel are shown in Figures 14 and 17.

\section{DERIVATION OF THE SHAPE OF THE CAVITY AND THE MOLD INSERT}

Due to the associativity between the 3D parametric model and the derived negative and positive shape of the mold parts, the functionality of publishing the geometry of the model, the so-called Publications. In such a case, the import of the whole Part is not required for the model of the cavity and the insert, but only the so-called
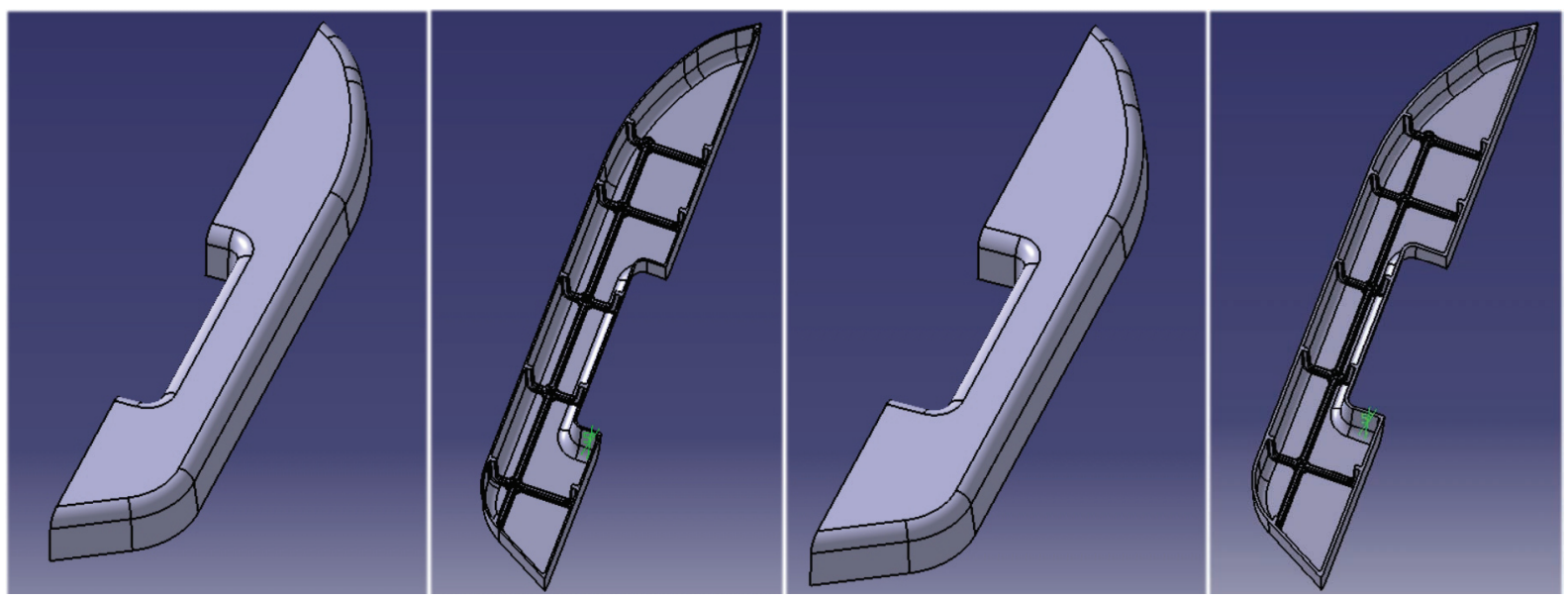

Fig. 14. Two different size model drive by parameters define and drive by Design Table 

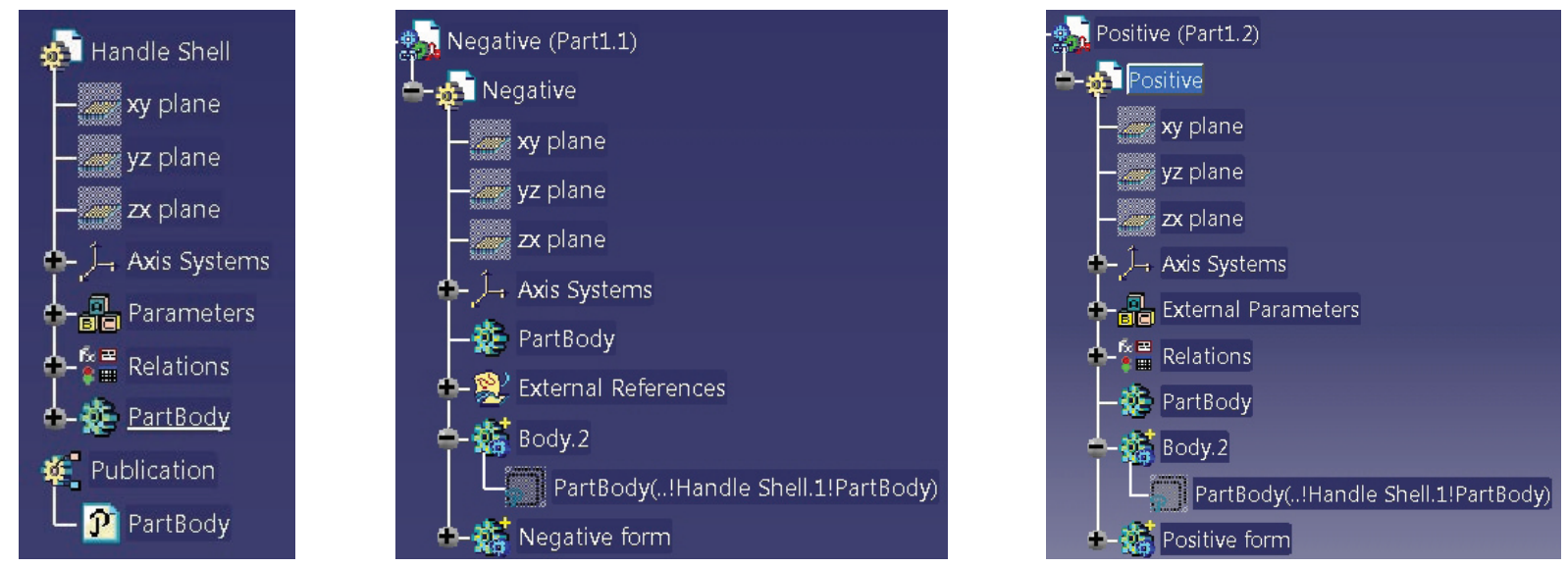

Fig. 15. Tree Product Handle Shell with Publications and example of trees derived parts Negative and Positive
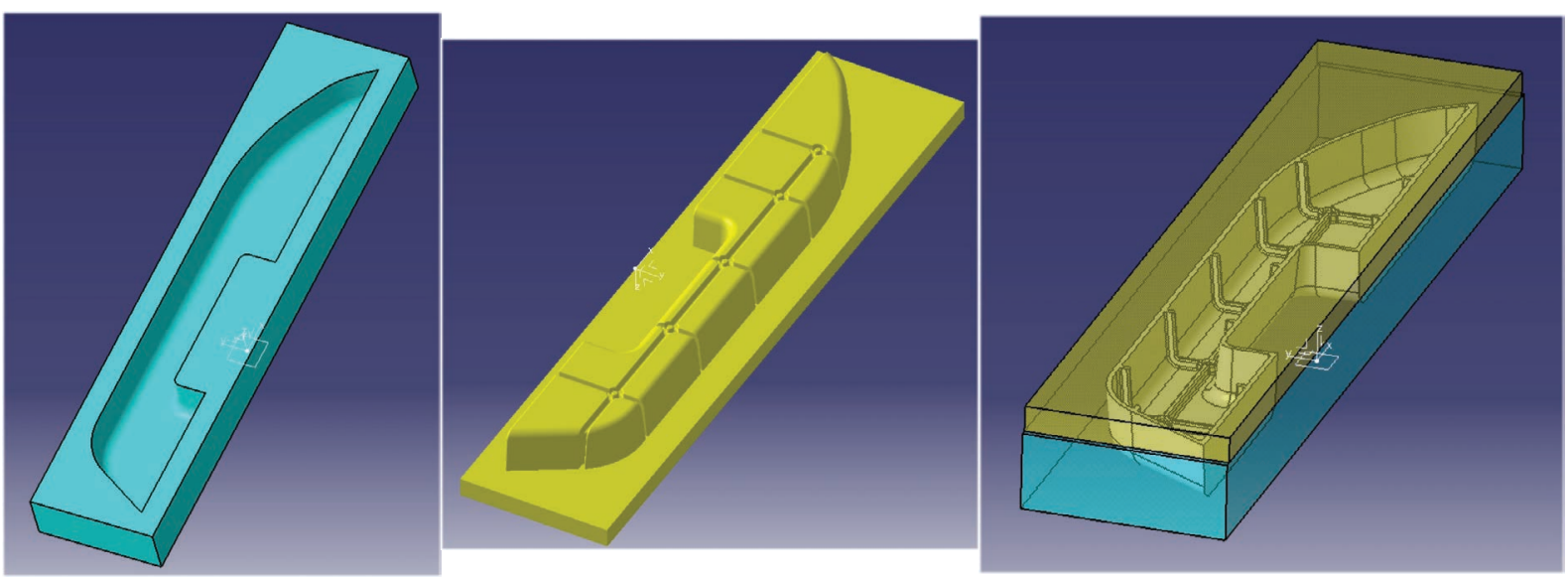

Fig. 16. Positive and Negative parts of Form, assembly of Mold Form

Publication (Fig. 15), which is a link to the original model. In this case, any change to the model will affect every other model where the Publications link appears. In Negative and Positive, the Publication is displayed as Solid.1. On the basis of the model, the shape of the cavity (Fig. 16 left) and the mold insert (Fig. 16 - middle) were derived by using Boolean Operations Remove and Remove Lump function.

\section{STL DATA GENERATION AND PRODUCTION USING 3D PRINTING TECHNOLOGY}

The geometric data from CATIA were exported to STL (Standard Triangle Language) format. The STL of the model as loaded into the Z-Suite software (Fig. 18), where the material (ABS) was defined, the printing strategy was determined and the parameters influencing the print quality and time were set (Fig. 19). On the basis of these settings, a control code was generated for the Zortrax M200 3D printer.

The model was set up in the printing space to achieve the best possible quality of the surface of the printed model. Due to the fact that the model is hollow inside and has different reinforcing ribs, it was necessary to print the supporting structures of the model, which were physically removed after printing. The model was printed at a reduced size. Printing took 6 hours when the print layer height was set to $0.19 \mathrm{~mm}$. The physical printout of the model is shown in Figure 20.

\section{CONCLUSION}

This paper demonstrates that a CAD model based on predefined parameters is flexibly 

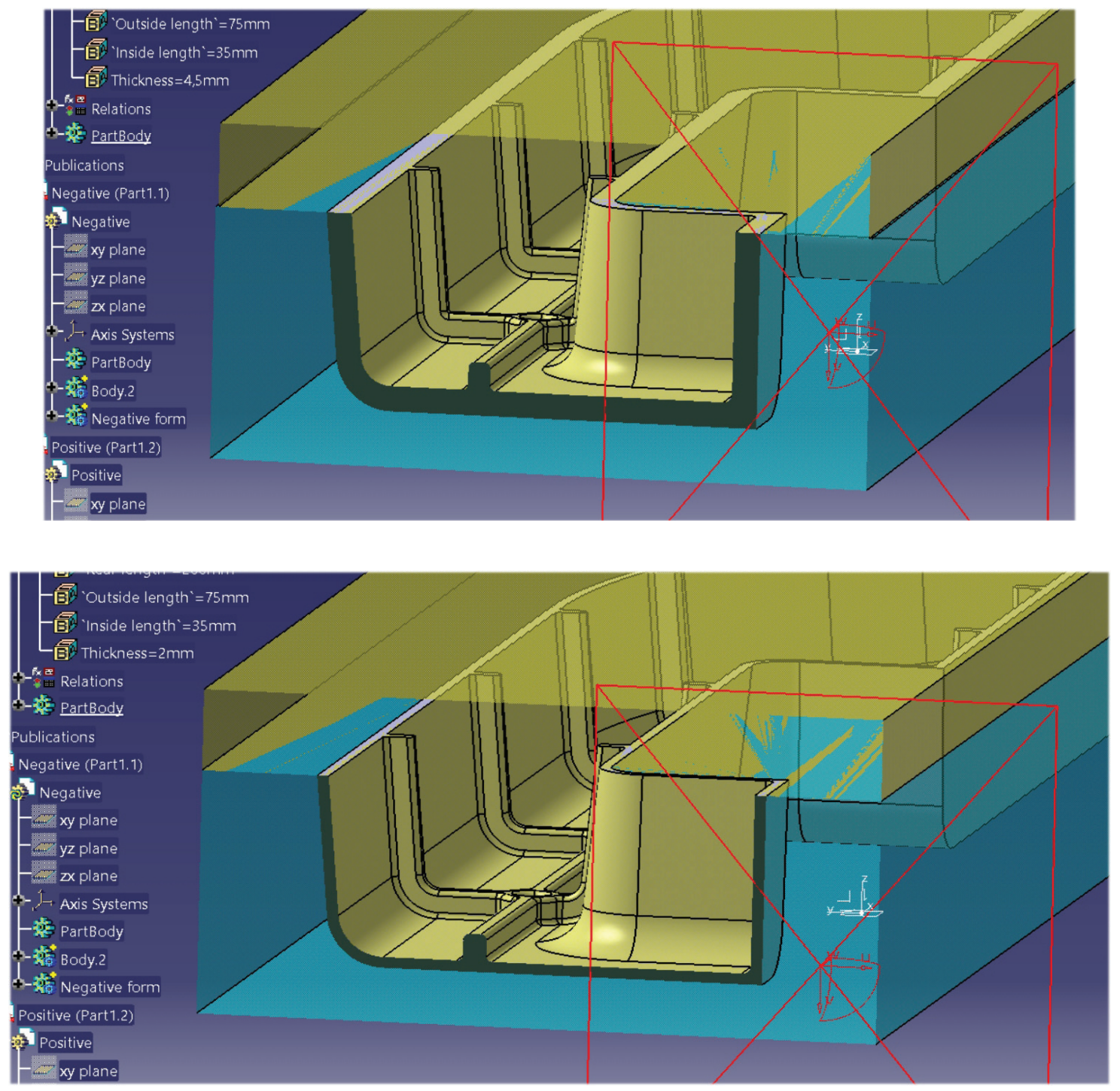

Fig. 17. Two different inside structures of Part and Form

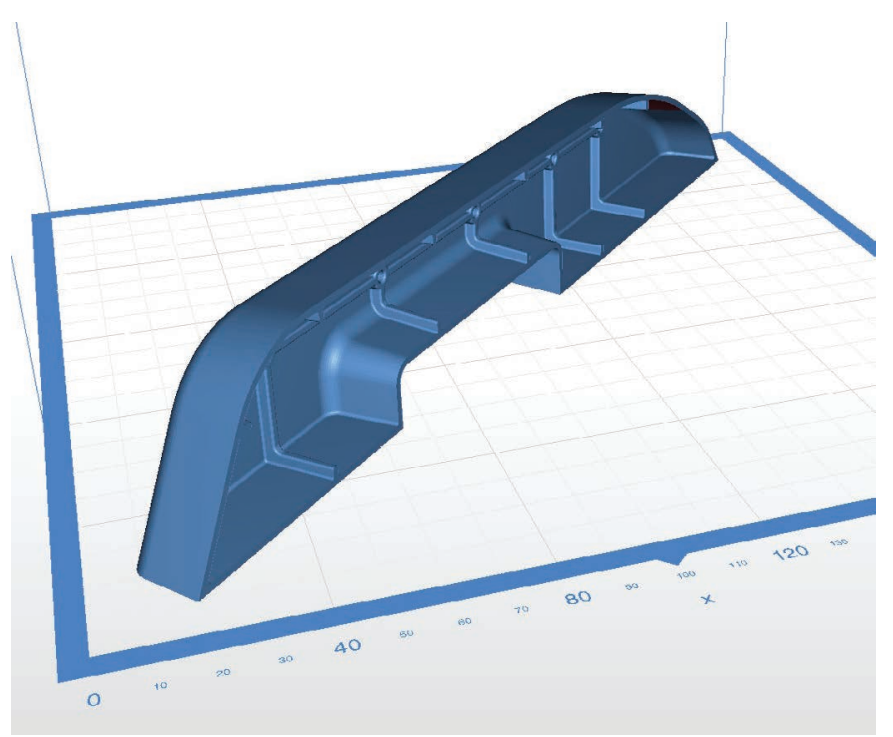

Fig. 18. STL format imported in the Z-Suite software 

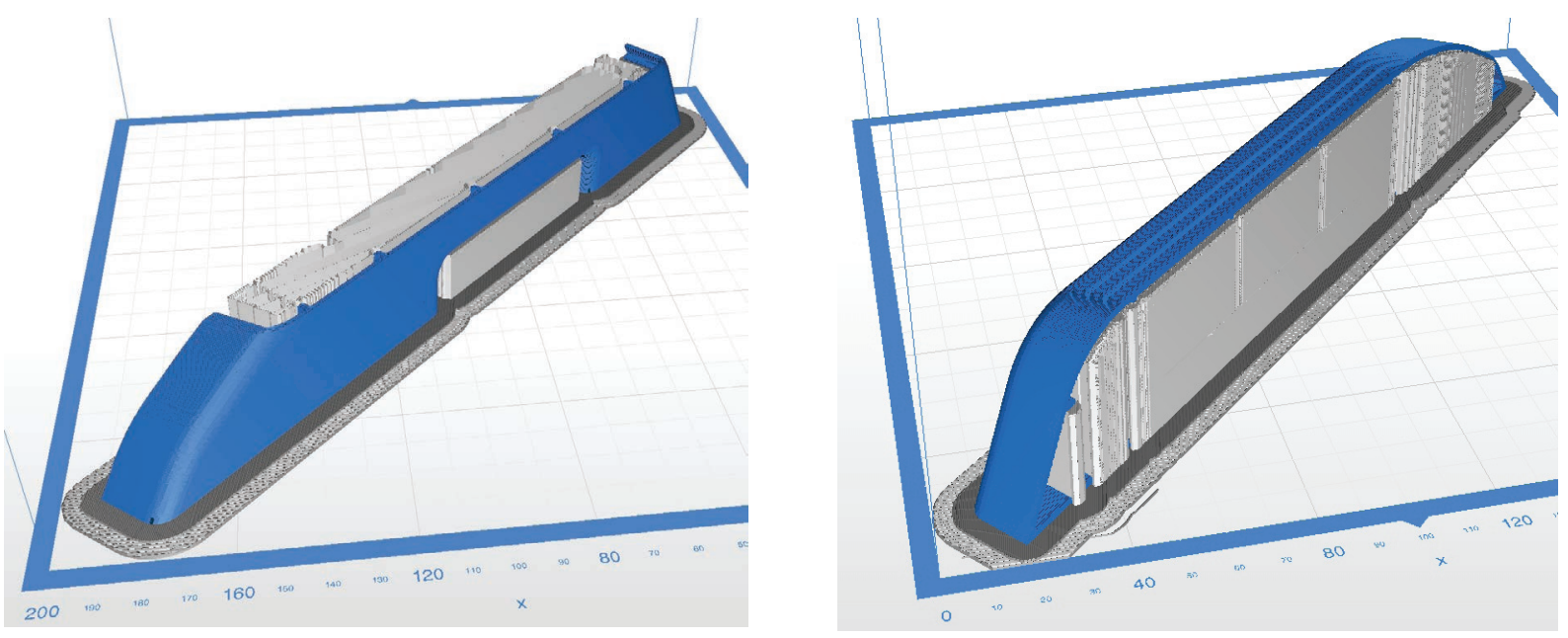

Fig. 19. Simulation of the model printing process in the Z-Suite software
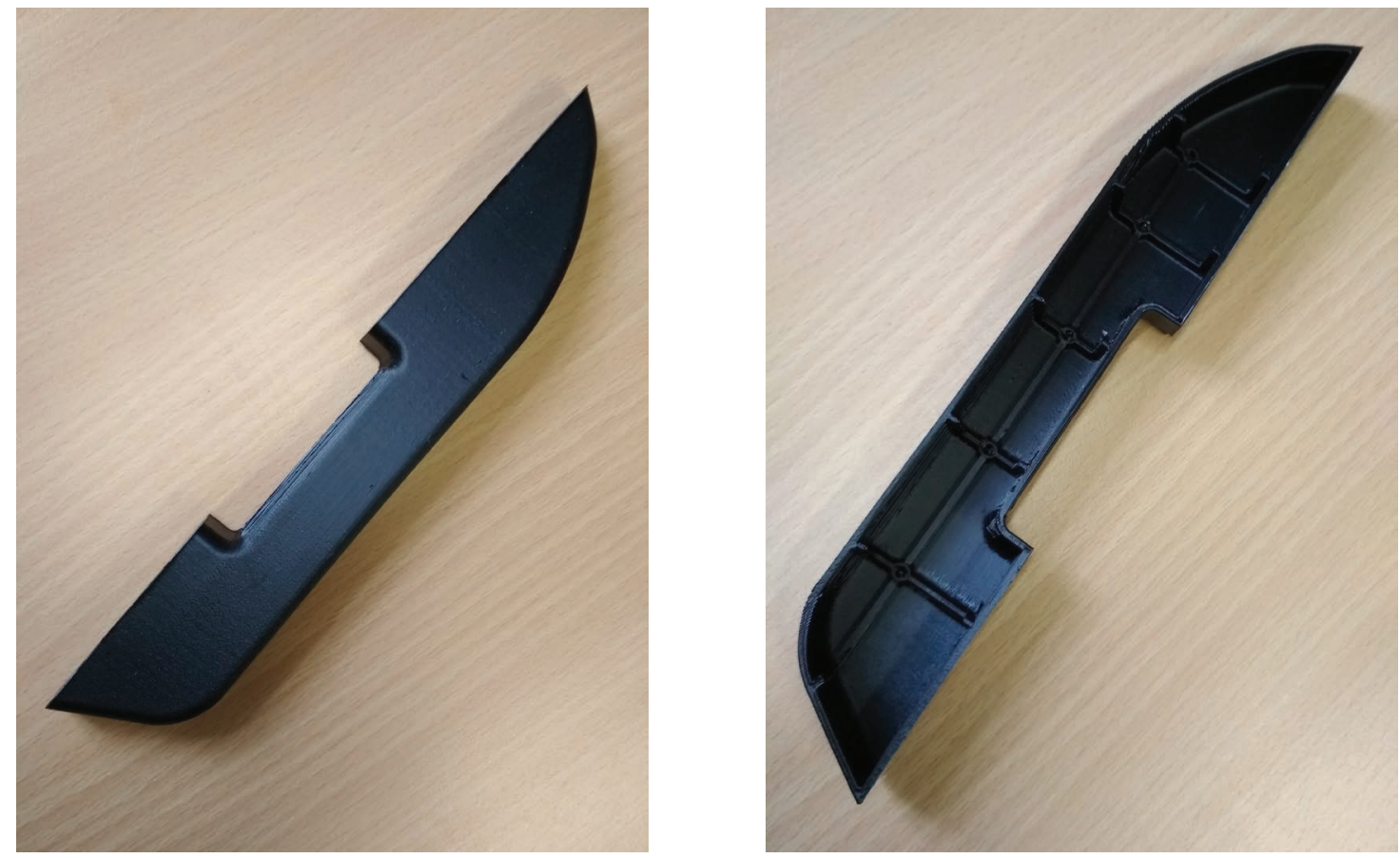

Fig. 20. Generating data for $3 \mathrm{D}$ printing and the $3 \mathrm{D}$ printed model

changeable in real time. Model control can still be “automated" by connecting a CAD system to a spreadsheet. An important advantage is that the changes in shape are reflected not only in the shape of the part but also in all assemblies where the reference to the part in the form of so-called "Publication" occurs.

As a result of the work, we confirmed that the parameterization of the model makes sense especially in the first stages of finding the shape of the model, when it is necessary to perform a number of repetitive operations that are necessary to achieve the result. Such an approach saves time and resources invested in product development.

\section{Acknowledgments}

This work was supported by VEGA $1 / 0110 / 18$ "Research and development in the area of reverse engineering and rapid prototyping focused on innovations of constructional parts designed for the experimental vehicles and transport equipment”. 


\section{REFERENCES}

1. Hirz, M., Harrich, A., Rossbacher, P.: Advanced computer aided design methods for integrated virtual product development processes. ComputerAided Design \& Applications, 8(6), 2011, 901-913.

2. Stadler, S., Hirz, M., Thum, K., Rossbacher, P.: Conceptual full-vehicle development supported by integrated computer-aided design methods. Computer-Aided Design \& Applications, 10(1), 2013, 159-172.

3. Schelkle, E., Elsenhans, H.: Virtual vehicle development in the concept stage-current status of CAE and outlook on the future. 3RD MSC Worldwide Aerospace Conference \& Technology Showcase, Toulouse, France, September 24-26, 2001.
4. Macey, S., Wardle, G.: H-Point, The fundamentals of car design and packaging. Design Studio Press, Culver City, www.designstudiopress.com

5. Daneshjo, N., Hajduova, Z., Dudas-Pajerska, E., Danishjoo, E.: Design of dedicated automated devices with CAD support. MM Science Journal, Mar. 2019, †2795-2799.

6. Fecova, V., Michalik, P., Zajac, J.: Up milling technology and its outputs, operation and diagnostics of machines and production systems operational states II. Book Series: †Applied Mechanics and Materials. Trans Tech Publications Ltd, 616, 7 , 2014P $\ 268-275$.

7. Czech, P., Turon, K., Barcik, J.: Autonomous vehicles. Scientific Journal of Silesian University of Technology-Series Transport, \100 P\15-22. 